\title{
Glass phenomenology in the hard matrix model
}

\author{
Junkai Dong, Veit Elser, Gaurav Gyawali, Kai Yen Jee, \\ Jaron Kent-Dobias, Avinash Mandaiya, Megan Renz, and \\ Yubo Su \\ Laboratory of Atomic \& Solid State Physics, Cornell University, Ithaca, NY \\ 14853-2501, USA
}

\begin{abstract}
We introduce a new toy model for the study of glasses: the hardmatrix model (HMM). This may be viewed as a single particle moving on $\mathrm{SO}(N)$, where there is a potential proportional to the 1-norm of the matrix. The ground states of the model are "crystals" where all matrix elements have the same magnitude. These are the Hadamard matrices when $N$ is divisible by four. Just as finding the latter has challenged mathematicians, our model fails to find them upon cooling and instead shows all the behaviors that characterize physical glasses. With simulations we have located the first-order crystallization temperature, the Kauzmann temperature where the glass would have the same entropy as the crystal, as well as the standard, measurement-time dependent glass transition temperature. Our model also brings to light a new kind of elementary excitation special to the glass phase: the rubicon. In our model these are associated with the finite density of matrix elements near zero, the maximum in their contribution to the energy. Rubicons enable the system to cross between basins without thermal activation, a possibility not much discussed in the standard landscape picture. We use these modes to explain the slow dynamics in our model and speculate about their role in its quantum extension in the context of manybody localization.
\end{abstract}




\section{Introduction}

Much of statistical mechanics is the study of "toy models," minimalistic distillations of physical systems that capture particular phenomena. The simplest model of liquids, mono-disperse hard spheres, is also much used as a model of glassy behavior. In three dimensions, and when compressed rapidly, this system produces jammed structures with a reproducible packing fraction [1, but without any obvious order. However, the hard sphere model falls short in exhibiting all facets of glass phenomenology and is difficult to treat analytically. We examine these shortcomings in connection with a new and even simpler model that might better serve as a model of structural glasses.

The hard matrix model (HMM) is a system comprising a single orthogonal matrix $U \in \mathrm{SO}(N)$ with 1-norm energy:

$$
\Phi(U)=-\sqrt{N} \sum_{i j}\left|U_{i j}\right| .
$$

The matrix elements are not independent, but constrained much like the bond lengths and angles in a network glass. Their number, $N^{2}$, is the "volume" of the system. The constraints in the hard sphere model are considerably weaker, and allow small clusters of spheres to act independently when they occur within a low density fluctuation. In fact, it is precisely such finite sized equilibrium fluctuations that make the hard sphere system unstable to nucleating the crystal phase If analogous nucleation events/structures exist for network glasses, they are poorly understood. The hard matrix model poses this same challenge, in a far simpler mathematical setting, because it too is potentially unstable to the analog of crystallization.

Matrix variables have previously been considered in models of glassy behavior without quenched disorder. To the best of our knowledge these have always relied on quartic potentials and fall short of the simplicity of our model (a standard norm imposed on a ubiquitous mathematical object). The square case $(\alpha=1)$ of the model studied by Cugliandolo et al 2 has only a temperature parameter, like ours, but no glassy properties when the variables are continuous, as in a physical network glass. While it is true that the ground states of model 2 when restricted to Ising $( \pm 1)$ variables coincide with the HMM ground states, we believe the anharmonicities in the continuous variable setting are just as important when modeling glassy systems. The models considered by Soljacić and Wilcek [3], with more parameters, are continuous but the proliferation of \pm 1 ground states is enabled by the element-wise anharmonicity $\left(1-U_{i j}^{2}\right)^{2}$, which defines a rather trivial energy landscape.

In some ways the HMM resembles a mean-field spin glass model: it lacks clear dimensionality, has "all-to-all" interactions, and the global orthogonality constraint resembles the spherical constraint of the spherical $p$-spin model 4, 5]. However, unlike most spin glasses the HMM has no quenched disorder and therefore a sampleindependent collection of equilibrium ground states, which we will see in the next section. At present the precise nature of its glassy behavior is not well-understood, and the status of hallmark spin-glass properties, such as the existence of a dynamical transition, is unknown [6. We hope that this paper inspires investigation into these aspects of the HMM.

In this paper we study several properties of the HMM. Section 2 describes the ground and low-temperature equilibrium states of the model, and the transition analogous to crystallization that leads to them. In Section 3 the focus shifts to

$\ddagger$ The same mechanism challenges other packing systems, e.g. tetrahedra, as candidate glass models. 
the main topic: the metastable glass phase of the model. Section 4 introduces a quantum generalization, its conjectured phase diagram, and thoughts about manybody localization. Finally, Section 5 summarizes our results, including the many advantages the HMM has over hard spheres.

\section{Equilibrium Thermodynamics}

By the generalized mean inequality we know

$$
\Phi(U) \geq-\sqrt{N} N \sqrt{\sum_{i j}\left|U_{i j}\right|^{2}}=-N^{2},
$$

where equality is attained only when the elements of $U$ are equal in magnitude. The ground states $U^{*}$ of $\Phi$ are therefore rescaled Hadamard matrices [7] $U^{*}=H / \sqrt{N}$, where $H$ has only \pm 1 elements, for those $N$ where Hadamard matrices exist. By contrast, the rigorous ground state characterization of the hard sphere model, the proof of the Kepler conjecture, required a massive amount of work [8]. There is also a Hadamard matrix conjecture, which asserts that Hadamard matrices exist for all orders $N$ divisible by four. Empirically, from explicit enumeration up to $N=32[9$, the number of Hadamard matrices \# $(N)$ [10] enjoys robust growth:

$$
\log \#(N) \sim 0.874 N^{1.6} .
$$

Ironically, for most (evenly-even) $N$ we lack even a single example [11, the smallest open case of the conjecture being $N=668$. It is for this reason that the hard matrix model still deserves to be called "hard." Simple physics-inspired methods, such as gradient descent on $\Phi(U)$ from random starting points, almost always fail at finding Hadamard matrices. The most successful methods for constructing these matrices [7 are algebraic in nature and require significant computation. However, because even the most productive of these are based on first finding sequences of size $N^{1}$ with special properties, the estimate (3) suggests that most Hadamard matrices are evading discovery.

Not only are the ground states of $\Phi$ known, so are the thermodynamic equilibrium states in the limit of zero temperature. To see this, parameterize the neighborhood of a ground state $U^{*}=H / \sqrt{N}$ with a skew-symmetric matrix $X$ :

$$
U(X ; H)=H e^{X} / \sqrt{N} .
$$

Expanding (11) for small $X$ we find

$$
\begin{aligned}
\Phi(X ; H) & =-\sum_{i j} \operatorname{sgn}\left(H_{i j}\right)\left(H\left(1+X+\frac{1}{2} X^{2}+\cdots\right)\right)_{i j} \\
& =-\operatorname{Tr}\left(H^{T} H\left(1+X+\frac{1}{2} X^{2}+\cdots\right)\right) \\
& =-N^{2}+\frac{N}{2} \operatorname{Tr}\left(X^{T} X\right)+\cdots,
\end{aligned}
$$

that is, the potential function reduces to a diagonal quadratic form independent of the ground state Hadamard $H$. That the contributions to the free energy are the same for all the ground states is in contrast to the analogous situation for hard spheres in three dimensions, where the free energy dependence on the stacking sequence of the close-packed triangular layers was discovered only recently and required elaborate computations [12]. 
Thanks to the simplicity of the local potentials (5), the low-temperature limit $\beta \rightarrow \infty$ of the HMM partition function

$$
Z(\beta)=\int d U e^{-\beta \Phi(U)},
$$

can be evaluated explicitly. With the standard scale convention, the group invariant measure for small $X$ of the parameterization (4) is

$$
d U=\prod_{1 \leq i<j \leq N} \sqrt{2} d X_{i j}
$$

Since for $\beta \rightarrow \infty$ exactly the same Gaussian integral arises around each Hadamard point of $\mathrm{SO}(N)$, we obtain

$$
Z(\beta) \underset{\beta \rightarrow \infty}{\sim} \#(N) e^{\beta N^{2}}\left(\frac{2 \pi}{\beta N}\right)^{N(N-1) / 4}
$$

for those $N$ where $\#(N)>0$.

In the absence of quantum mechanics the entropy has an arbitrary additive constant and we are free to set $S(0)=0$. This is equivalent to working with the rescaled partition function $\bar{Z}(\beta)=Z(\beta) / Z(0)$ and defining the entropy by $S=\log \bar{Z}+\beta\langle\Phi\rangle$, where $\langle\cdot\rangle$ is the Gibbs average. Using the known volume of $\operatorname{SO}(N)$ [13,

$$
Z(0)=\int d U=2^{(N-1)(N / 4+1)} \prod_{k=2}^{N} \frac{\pi^{k / 2}}{\Gamma(k / 2)},
$$

we then have an explicit expression for the HMM entropy in the low temperature limit. Taking additionally $N$ large, as in a thermodynamic limit, we obtain the entropy per volume for $\beta \rightarrow \infty(e=2.718 \ldots)$ :

$$
s=\frac{1}{N^{2}} S \underset{N \rightarrow \infty}{\sim}-\frac{1}{4} \log (2 \beta \sqrt{e})+\frac{1}{N^{2}} \log \#(N) .
$$

This result looks like it might be used to address the Hadamard matrix conjecture. By integrating the HMM specific heat $c(\beta)$ in a Markov chain Monte Carlo (MCMC) simulation, starting at $\beta=0$, it should be possible to obtain reasonable estimates of $s(\beta)-s(0)=s(\beta)$. A comparison with (10), while lacking the precision to determine $\#(N)$ outright, might still give information about its growth. As we show next, this works for small $N$. However, large $N$ is inaccessible, and the Hadamard matrix conjecture remains safe, for the same reasons that make the HMM compelling as a model for glass. Cugliandolo et al 2] noticed that the \pm 1 case of their matrix model has Hadamard (for $\alpha=1$ ) ground states. However, in the continuous ("spherical") case of this model the ground state is just the $\mathrm{SO}(N)$ manifold and not glassy in the least. Our model has the important advantage that the hard-to-find ground states are also embedded in a non-trivial continuum landscape.

Among glass models the HMM is relatively easy to simulate. We sampled the Gibbs ensemble using MCMC, with elementary transitions generated by Givens rotations applied to pairs of rows and columns of $U$. The range of the Givens angle was tuned, at each temperature, so the resulting acceptance rate is $50 \%$. By defining a "sweep" of the system to be rotations attempted on all pairs of rows and columns, a single MCMC sweep is a reasonable proxy for a time step of true dynamics, since the number of actual moves per sweep scales with the number of continuous degrees of freedom. MCMC simulation of our model's equilibrium thermodynamics benefits from 
the use of parallel tempering, and we use this method when the stable equilibrium is achievable and simulation time scales are not our interest. When parallel tempering was used, as for the results of Figure 1. temperatures were selected to optimize the replica transit time 14 .

Figure 1 shows the MCMC specific heat, or heat capacity per unit volume, $c=C / N^{2}$, for $N=12,16,20$. The evidence for a first-order phase transition in the infinite system is strong. We will say the system at high temperatures is in the liquid phase, and at low temperatures in the Hadamard phase. The latter is in fact a collection of $\#(N)$ phases, each associated with a different Hadamard "crystal" ground state. At $N=20$ we are already at the limit of being able to maintain thermal equilibrium with Givens rotations. Near the specific heat peak, MCMC simulations require $2 \times 10^{9}$ sweeps per measurement, while precise measurement by parallel tempering took $1 \times 10^{8}$ sweeps per temperature with $2 \times 10^{7}$ adjacent replica steps. A good test of the accuracy of the $c(\beta)$ curve is the corresponding entropy integral. When compared against (10), this reproduced the known Hadamard count $\#(20) \approx 2 \times 10^{45} \quad 10$ to within a factor of 1.28 , a $0.06 \%$ error in the entropy The low temperature limit $c=1 / 4$ is simply the equipartition value $1 / 2$ for quadratic potentials combined with the number of continuous modes being only half the system volume.

The HMM challenges our understanding of first order phase transitions. Consider the notion of phase coexistence. We suspect that the HMM does not exhibit phase coexistence in the usual sense. Suppose the system is prepared with energy density halfway between that of the pure phases at the transition, say by MCMC sampling at the temperature of the specific heat peak (in a modest sized system where this is possible). What might such a system look like? We doubt that the configuration will be mixed-phase in the usual sense, say a proper Hadamard submatrix within a "liquid matrix." If such mixed configurations existed, with continuously variable composition, then it would also be possible to have critical nuclei for crystallization, contrary to the extreme degree of metastability we observe already for $N=24$.

While all of our numerical experiments used MCMC, true dynamics could be simulated by time-evolving the unconstrained system (see Appendix)

$$
\begin{aligned}
\mu N\left(\ddot{U}+\dot{U} \dot{U}^{T} U\right) & =-\frac{1}{2}\left(\nabla \Phi-U(\nabla \Phi)^{T} U\right) \\
& =\frac{\sqrt{N}}{2}\left(\operatorname{sgn}(U)-U \operatorname{sgn}(U)^{T} U\right),
\end{aligned}
$$

with initial constraints

$$
U(0) U^{T}(0)=1, \quad \dot{U}(0) U^{T}(0)+U(0) \dot{U}^{T}(0)=0 .
$$

The left-hand side of (11) generates free motion on $\mathrm{SO}(N)$, and the scaling of the mass with $N$ was chosen so the equations for small oscillation about the Hadamard minima,

$$
\mu \ddot{X}=-X,
$$

are independent of $N$. From (12) we see that the mechanical equilibrium points of $\Phi(U)$ correspond to orthogonal matrices with the following symmetry property:

$$
U^{T} \operatorname{sgn}(U)=\operatorname{sgn}(U)^{T} U
$$

$\S$ This is before making large $N$ approximations, as the Hadamard count makes a subextensive contribution. The counts in OEIS A206711, which include Hadamards of negative determinant, were divided by two. 

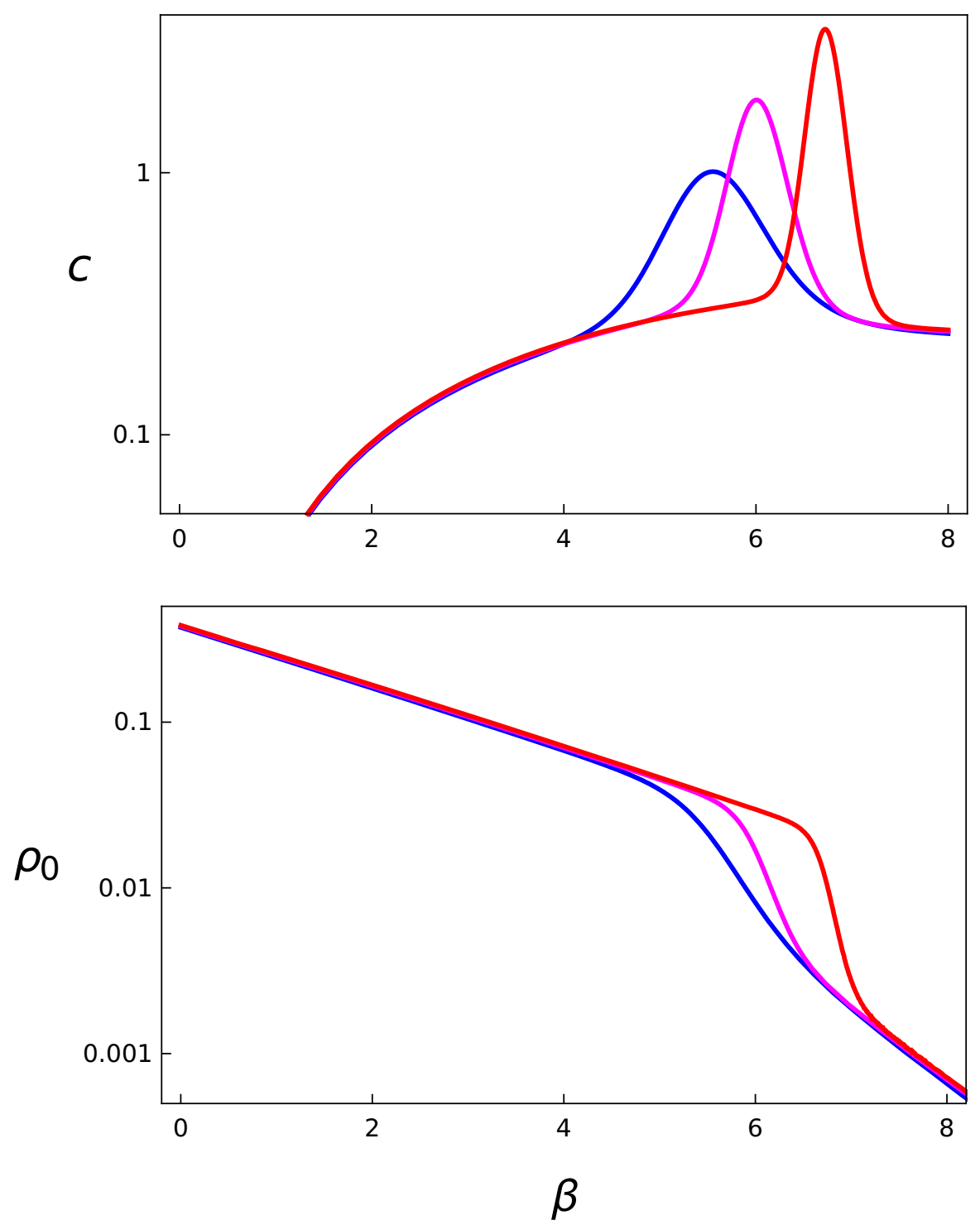

Figure 1. Equilibrium specific heat (top) and transition state density (bottom) for system sizes $N=12$ (blue), 16 (magenta), 20 (red). Narrowing of the specific heat peak and abruptness in the drop of $\rho_{0}$, with increasing $N$, indicate a first order transition. The vertical axis on both plots is logarithmic, and the statistical errors at the sample points are smaller than the thickness of the curves. Comparison of the entropy change defined by the numerical integral of the specific heat agreed with the results of direct Hadamard matrix enumeration to within $0.06 \%$ 
These are a superset of the Hadamard matrices and it is their high abundance that defeats the prospect of finding Hadamard matrices by gradient descent on $\Phi(U)$. It is tempting to look at property (15) as a set of geometrical constraints of exactly the right number to fix all the continuous variables of an orthogonal matrix, in analogy with isostaticity in jammed sphere packings or rigidity of ball-and-stick network models. While this perspective can be useful for identifying good glass formers when constituents are modeled geometrically [15, in our case it is simply an automatic consequence of a sufficiently well-behaved potential function.

The mechanical equilibrium points are relevant for the dynamics at low temperature. Figure 2 shows a detail of $\rho(U)$, the distribution of the individual matrix elements, near $U=0$ where their contribution to the energy is highest. This distribution was generated by gradient descent from random points on $\mathrm{SO}(32)$. The property $\rho(0)>0$, which seems to hold in the thermodynamic limit, confers a fragility to the mechanical equilibria. Consider the set of matrix elements whose values are within some fixed, small distance of zero. For each of these there is a small geodesic motion that brings the matrix element to the transition state point of its energy, the cusp at $U=0$. These single-element transition states are likely also transition states for the system as a whole because the regular contribution to $\Phi$ (from the other matrix elements) only changes quadratically and the motion is small. Unlike phonon modes, for which the perturbation sees an opposing/restoring force, the opposite is true when a matrix element crosses $U=0$. These rubicon modes, present at all temperatures with density proportional $\rho(0)$, provide a mechanism for the system to sample the energy landscape without ever having to surmount an energy barrier. As we will see, the growth of the dynamical mixing time at low temperatures may not be the result of activated processes at all, but a byproduct of a sharply reduced rubicon density.

We believe the transition state density $\rho_{0}=\rho(0) / \sqrt{N}$ is the key structural property of our model. It is known that $\rho(U)$ is normally distributed at $\beta=0$ in the large $N$ limit [16, and yet as shown in Figure 3, it is strongly bimodal already at $\beta=3$. As in physical glass formers, the liquid phase of the HMM is strongly correlated at temperatures well above crystallization, and the smallness of $\rho_{0}$ is a useful measure of this. The first-order nature of the liquid/Hadamard phase transition is clearly seen in the discontinuity-tending behavior of $\rho_{0}$, with increasing $N$, shown in Figure 1 for the same system sizes discussed earlier. Finally, by being linked to dynamics via fragile equilibria and rubicon modes, $\rho_{0}$ will also be relevant to the discussion of glasses to which we turn next. In brief, we find $\rho_{0}$ has a simple Arrhenius behavior in the glass, much like the concentration of defects in a crystal. The onset of slow equilibration in glasses might therefore be less a consequence of rising energy barriers and more the result of a scarcity of modes that can stir the system. In the Hadamard phase $\rho_{0} \sim \sqrt{\beta / \pi} e^{-\beta}$ also has the Arrhenius form, but with a steeper slope than in the glass. This quantity is not linked to transition states (rubicons) in the Hadamard phase because, unlike the fragile equilibria, it becomes small simply by the suppression of the large thermal fluctuations required to create zero matrix elements.

\section{Metastable glass}

Much of glass phenomenology [17, 18] is captured in the series of specific heat measurements shown in Figure 4 for the $N=32$ system. These were initialized in the liquid phase and show no sign of an anomaly at the expected crystallization temperature, even at the longest equilibration times, or number of sweeps $t$. We 


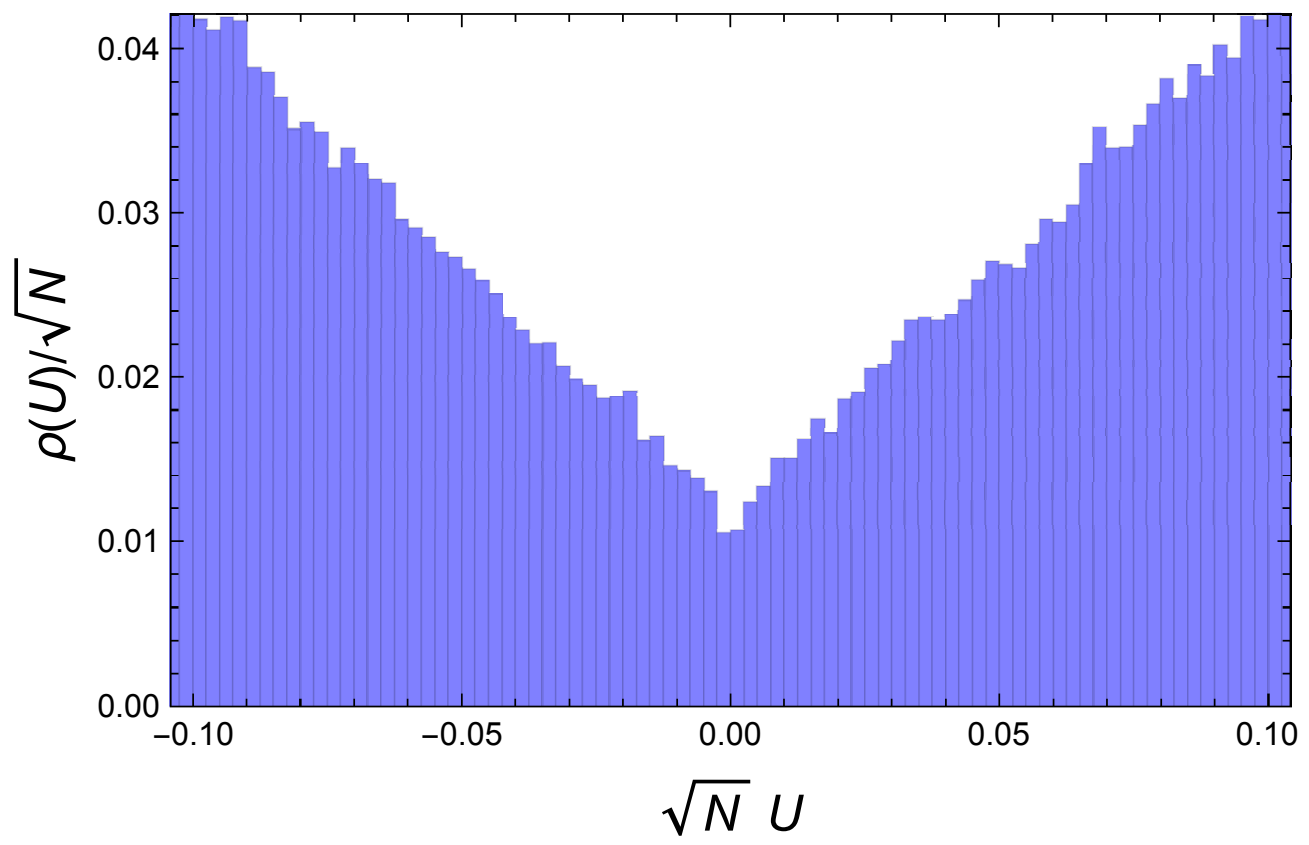

Figure 2. Detail of the distribution of matrix elements, near $U=0$, of equilibrium points generated by gradient descent. All equilibria are fragile in the sense that some fraction of the matrix elements are near a "transition state" in their contribution to the energy.

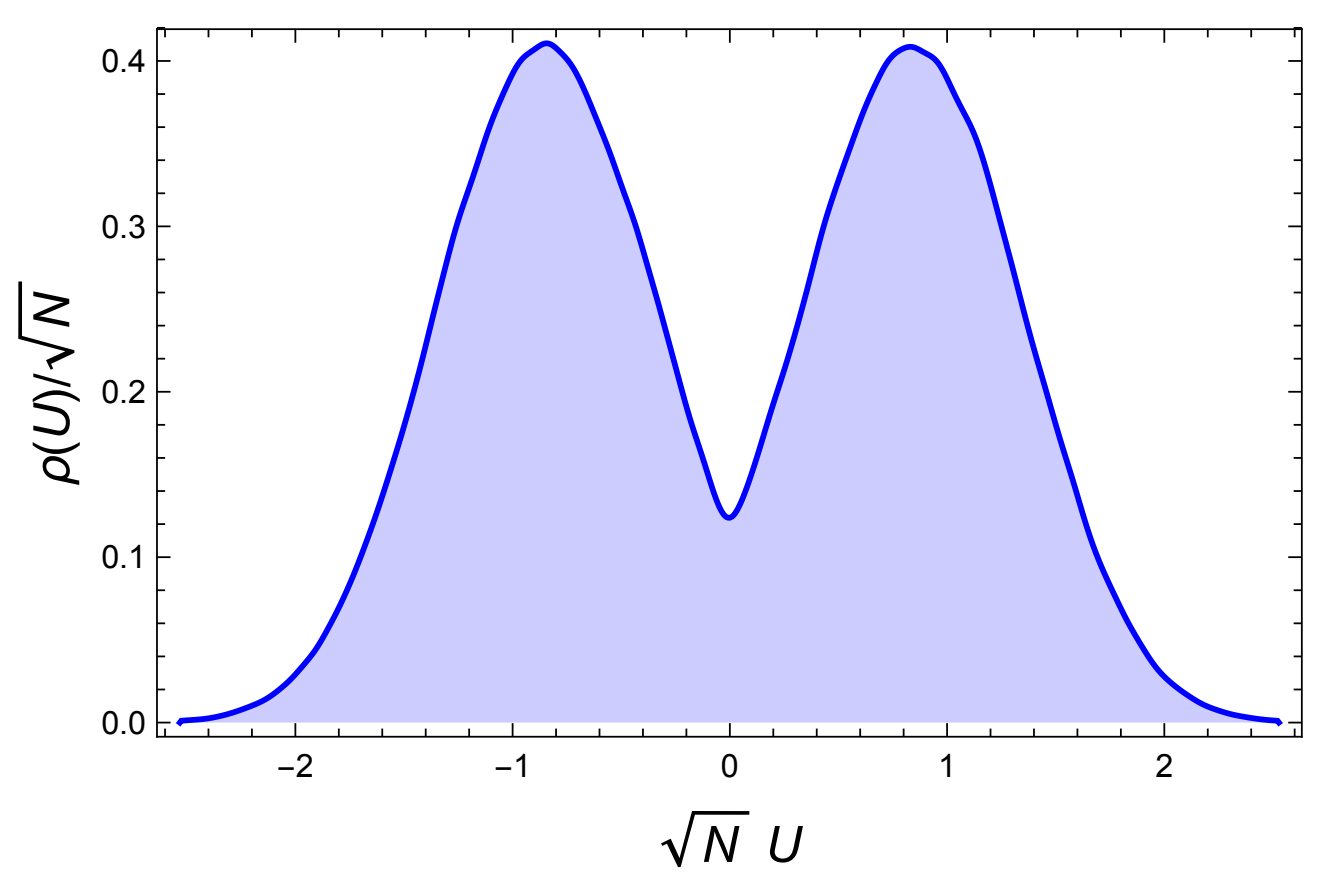

Figure 3. Probability distribution of the matrix elements at $\beta=3$, from a $N=32$ simulation, shows the liquid is strongly correlated at temperatures well above the phase transition. 


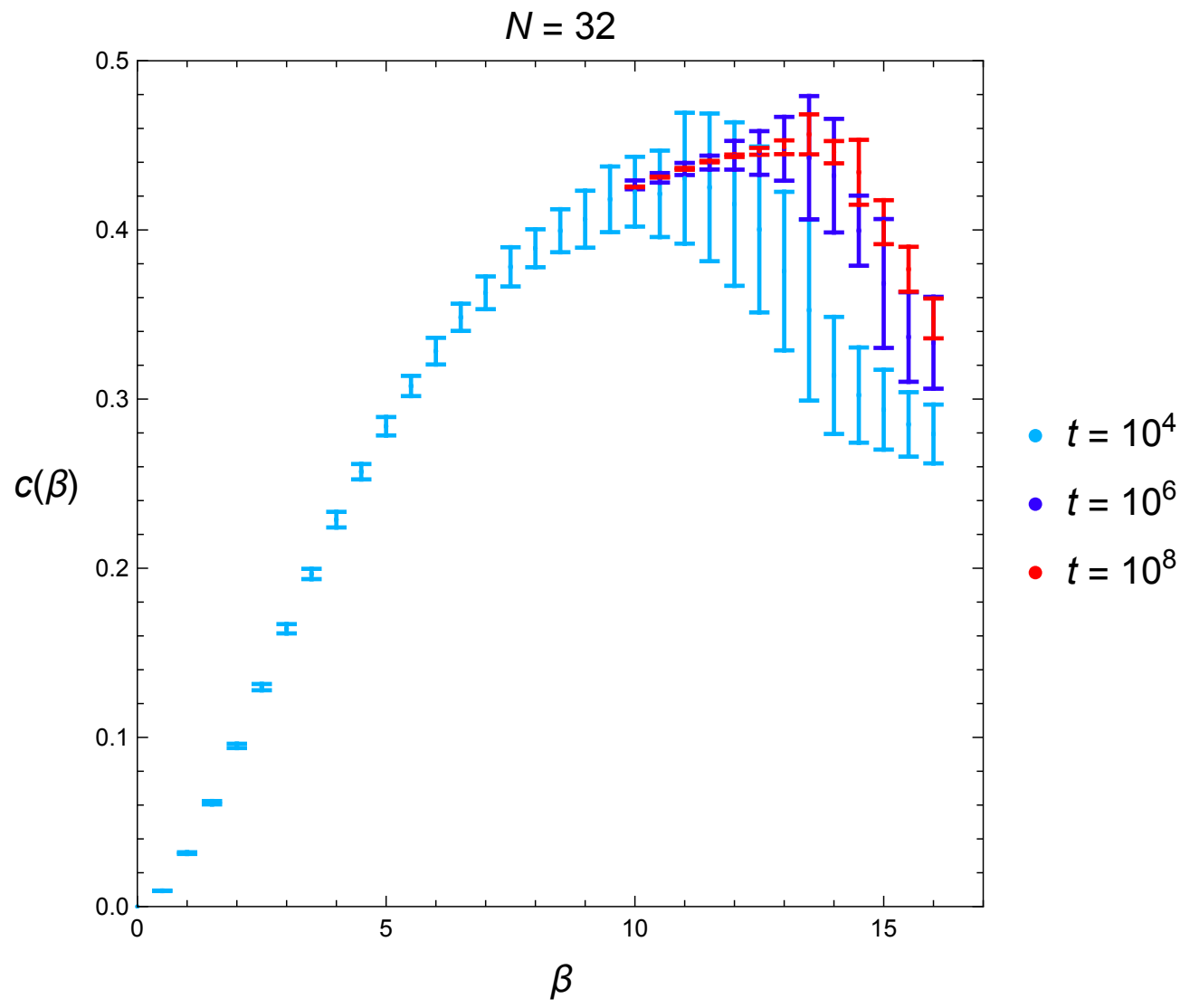

Figure 4. Specific heat of the $N=32$ system with increasing number of MCMC sweeps $t$ per measurement. The error bars show one standard deviation in the measurements of 20 systems.

estimated $\beta_{H}$ for $N=32$ by measuring the free energies of the metastable liquid and Hadamard phases by cooling the former and heating the latter. The free energy crossing is shown in Figure 5 and locates the transition at $\beta_{H}=7.9$. Instead of a peak in the specific heat at $\beta_{H}$, the cooled $N=32$ liquid exhibits a gently rising $c(\beta)$, with each reduction in cooling rate hopefully revealing a better equilibrated metastable phase: the HMM glass.

Upon closer examination of Figure 4 we see that there are actually two distinct phenomena. First, the fact that the drop in $c(\beta)$ shows signs of recovering with increasing $t$ can be interpreted by the system falling out of equilibrium: on the low temperature side of the broad specific heat peak the equilibration time is insufficient for the system to sample the full, equilibrium range of energy fluctuations. The second phenomenon concerns the error bars. These show one standard deviation in the measurements of 20 systems. We see a relatively abrupt transition between a regime where increasing $t$ has the desired $\sim 1 / \sqrt{t}$ convergence to a unique average, to another regime, at low temperature, where increasing $t$ has very little effect and instead it is the systems that have become unique.

The two phenomena we see in the glass phase specific heat are disentangled by 


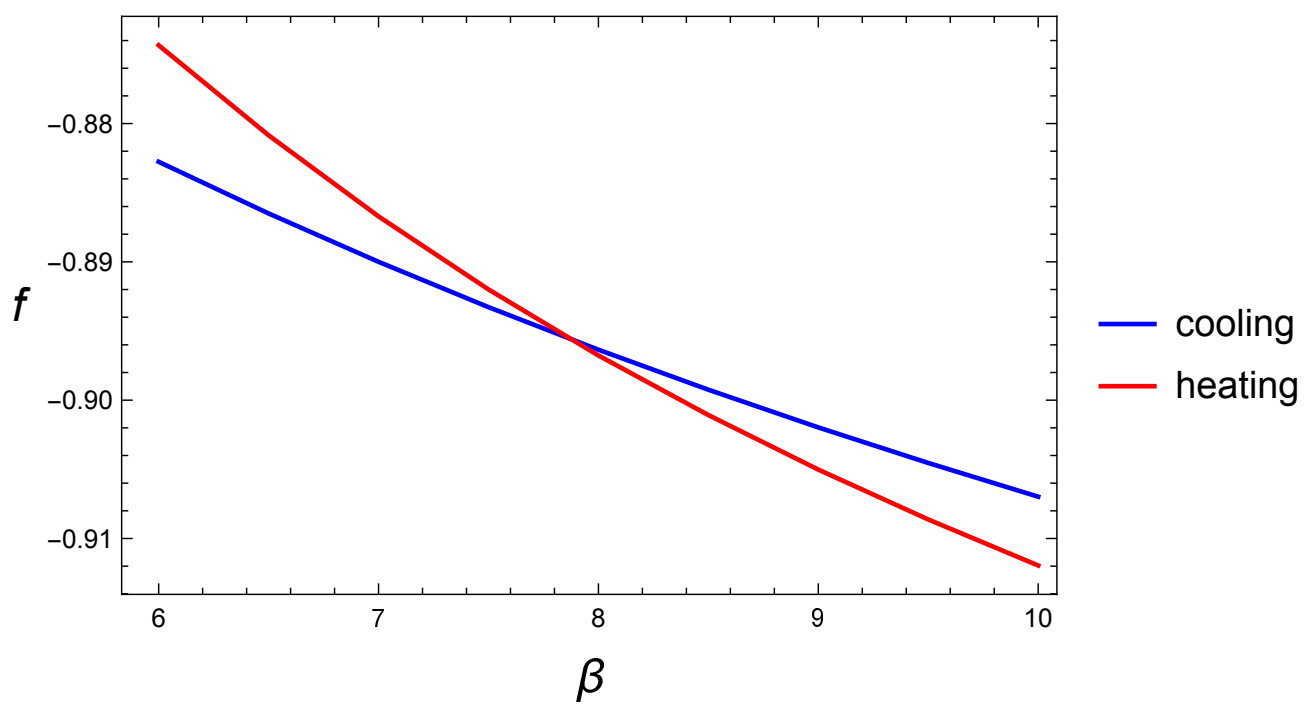

Figure 5. MCMC free energies of the $N=32$ system cooled from the liquid and heated from one of the crystal phases.

examining the time evolution of the correlation

$$
q(t)=\frac{1}{N} \operatorname{Tr} U^{T}(0) U(t) .
$$

As in the specific heat measurements, one unit of the time $t$ is a single MCMC sweep (with Givens rotations tuned to have $50 \%$ acceptance rate), and $10^{6}$ sweeps were used to equilibrate $U(0)$. Figure 6 shows the decay of $q(t)$ with time evolution, for four temperatures and three system sizes. Each experiment was repeated five times.

The results for the largest system size, $N=128$, are consistent with there being no dynamical transition. As $\beta$ increases, the correlation $q(t)$ has the same decay after a suitable rescaling of the time. However, the results also show that one might be led to a different conclusion when the thermodynamic limit $(N \rightarrow \infty)$ is not taken into consideration. For example, just based on the results for $N=64$ one might conclude there is a transition to a phase with "persistent history" for $\beta$ greater than about 14 .

The onset, at large $\beta$, of dynamics dictated by events in the distant past, can be explained using the transition state density $\rho_{0}$. We first observe, as shown in Figure 7. that $\rho_{0}$ has a simple Arrhenius thermal behavior:

$$
\rho_{0}(\beta) \propto e^{-\beta e_{0}},
$$

where $e_{0} \approx 0.46$. For both of the system sizes shown, the range in values from 20 experiments is smaller than the plot symbols already at $10^{4}$ sweeps and about 10 times smaller than this when the average is over $10^{6}$ sweeps. The plot also confirms that the density $\rho(U)$, with $U$ scaled by $\sqrt{N}$, is a thermodynamic density, independent of $N$, all the way to the $U=0$ transition-state elements. At the same time, the Arrhenius behavior of $\rho_{0}$ challenges the achievability of the thermodynamic limit in our model. If we assume that rubicon modes are the dominant mixing mechanism, then we should expect equilibrium in a thermodynamic sense only if the total number of rubicon modes is $O\left(N^{2}\right)$, and a breakdown occurs when $N^{2} \rho_{0}(\beta) \sim c$ for some $c=o\left(N^{2}\right)$. For example, taking $c=10$ and using (17), we should expect thermodynamic-limit 


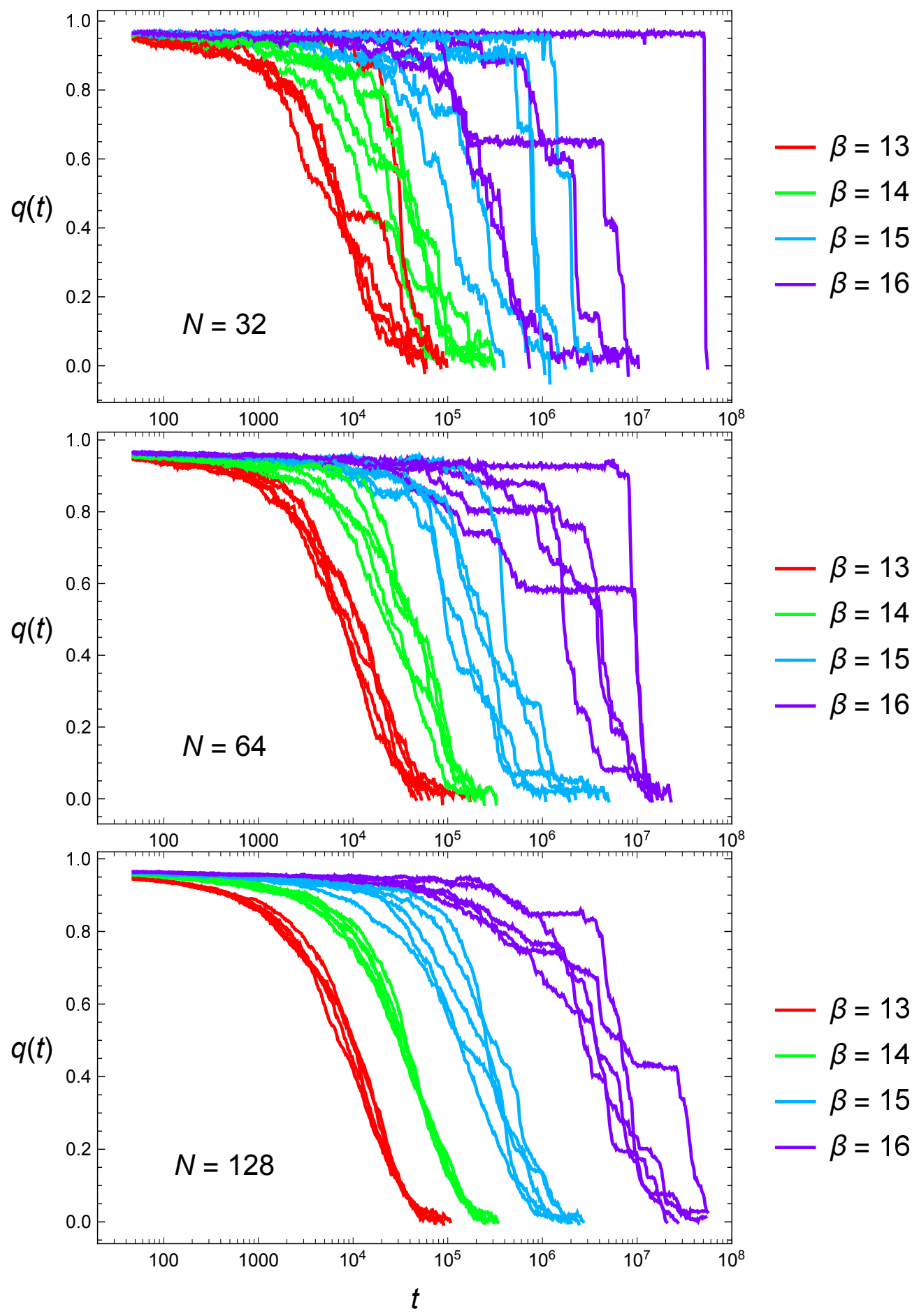

Figure 6. Time evolution of the correlation (16) for different inverse temperatures and system sizes. Equal increments in $\beta$ translates $q(t)$ along the logarithmic time axis by increasing amounts. Reproducibility of the corresponding time-rescaling symmetry, over the five experiments at each $\beta$, holds only when the system size is sufficiently large. 


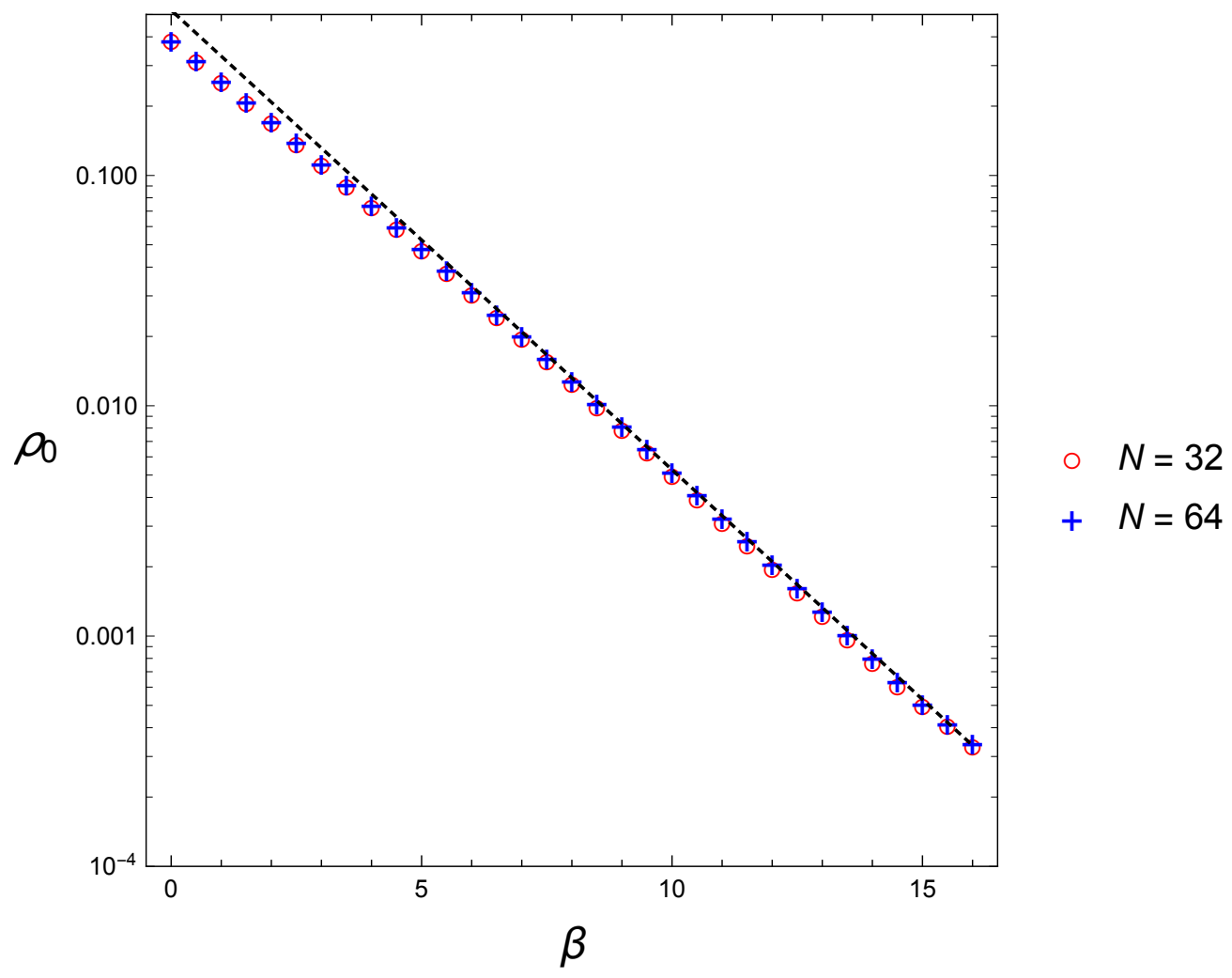

Figure 7. Arrhenius behavior of the transition state density $\rho_{0}$ for two system sizes. Shown are results for averages over $10^{6}$ sweeps; results with only $10^{4}$ sweeps are practically indistinguishable. The dashed line is a linear fit at the low temperature end.

behavior only for $\beta$ below 8.7, 11.7 and 14.7, respectively, in the $N=32,64,128$ systems.

We now turn to the problem of estimating the mixing time $\tau(\beta)$, that is, the timerescaling which collapses the the correlations $q(t)$ onto a single curve. As we argued above, this is an academic exercise in asymptotics since the result may hold only when the system volume is allowed to grow exponentially with inverse temperature. Our derivation makes a number of leaps in logic and is mostly an attempt to interpret an asymptotic form that empirically collapses the data despite having just a single parameter.

The main ingredient of the estimate is the idea that the number of independent modes in the mixing dynamics is proportional to the number of rubicons in the system, $\rho_{0} N^{2}$. Alternatively, we can think of the accessible states as lying in a space of dimension

$$
D(\beta) \propto \rho_{0}(\beta) N^{2}
$$

within $\mathrm{SO}(N)$ and on which there is free motion. If $\xi(\beta)$ is the distance scale (on $\mathrm{SO}(N)$ ) associated with each rubicon mode, then a sufficient condition for mixing is the volume-of-states criterion

$$
\xi(\beta)^{D(\beta)} \sim e^{S_{0}},
$$




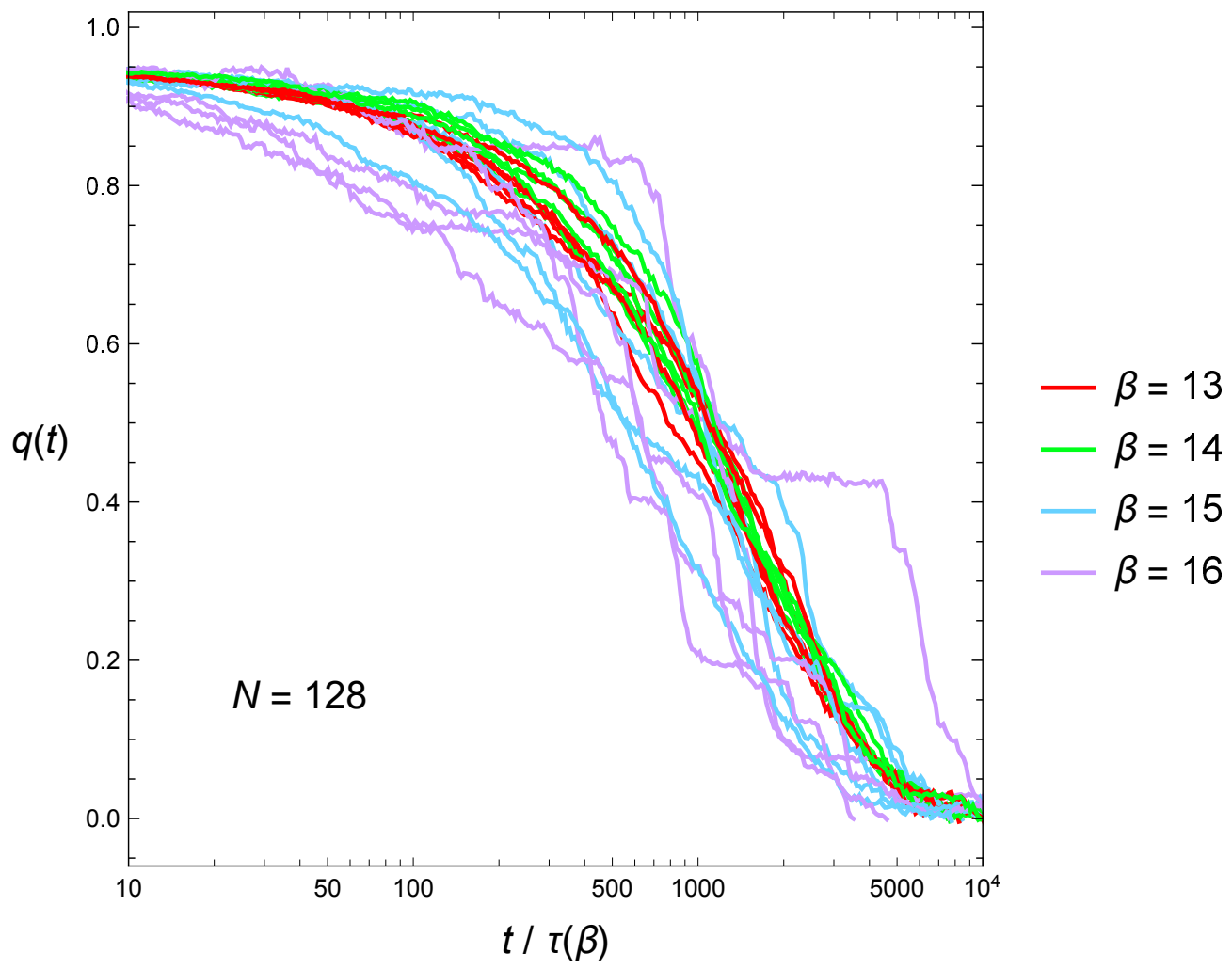

Figure 8. Collapse of the $q(t)$ curves in Figure 6 for $N=128$, upon scaling the time by the mixing time 21] with $b=5.2$.

where $S_{0}=O\left(N^{2}\right)$ is an assumed temperature-independent entropy of the glass (conventionally designated "configurational"). The nature of the glassy dynamics, as expressed in this relation, is that the rubicon mode amplitudes $\xi$ are forced to be very long as the dimension $D$ becomes small at large $\beta$. Assuming diffusive rubicon motion, the mixing time enters by the relation

$$
\xi(\beta) \propto \sqrt{\tau(\beta)} .
$$

Combining (17), (18), (19) and (20) results in the large- $\beta$ behavior

$$
\tau(\beta) \propto \exp \left(\exp \left(\beta e_{0}-b\right)\right)
$$

with a single undetermined parameter $b$. The collapse of the $q(t)$ curves for the $N=128$ system is shown in Figure 8 for $b=5.2$ and with 1 as the proportionality factor in (21).

If we fix a proportionality factor $a$ in (21) so that $q(\tau)=1 / 2$ and designate this $\tau$ as the "measurement time" in an experiment, then solving (21) for $\beta$ defines the glass transition (inverse) temperature:

$$
\beta_{g}(\tau)=(\log (\log (\tau / a))+b) / e_{0} .
$$

The parameter $a$ depends on the details of the dynamical equations. For our 50\%acceptance-Givens-rotation dynamics, $a \approx 1000$. 
After the exercise of analysing the behavior of the correlations $q(t)$, in time as well as system size, we are in a better position to interpret the specific heat results of Figure 4 The default interpretation is that by the application of extraordinary resources, not just in measurement time but also system size, the specific heat will reveal itself to saturate at some value $c_{g}$ greater than the $c=1 / 4$ from the harmonic vibrations in the Hadamard phase. This predicts that the entropy of the glass at low temperatures has the form

$$
s_{g}(\beta)=-c_{g} \log \left(\beta / \beta_{0}\right),
$$

where $c_{g} \approx 0.46$ from the $N=32$ simulation and $\beta_{0} \approx 3.63$ comes from integrating the non-constant part of the specific heat. Comparing (23) with (10) we find that the Hadamard ("crystal") entropy would exceed the glass entropy for $\beta>\beta_{K} \approx 26$. This state of affairs is known as the Kauzmann paradox [19 and the $\beta_{K}$ so defined is the inverse Kauzmann temperature. If we take our measurement time estimate above seriously, then to sample such a paradoxical glass configuration a simulation would require of order $10^{400}$ time steps. We note that the abundance of Hadamard matrices - each a distinct "crystal" phase - does not resolve the paradox because this contribution to the entropy is non-extensive.

To resolve the Kauzmann paradox in our system one should not overlook the possibility that the specific heat has a maximum, even when the system is fully equilibrated. Provided $c(\beta)$ has a suitable approach to the Hadamard value $c=1 / 4$ at large $\beta$, the glass entropy can remain above that of the ordered Hadamard phase. The only thing odd about this scenario would be the somewhat large $\beta$ of the specific heat maximum. But even this can be dismissed because the known transition to the stable low temperature phase, $\beta_{H}$, is also large.

On a more fundamental level one might ask whether there is even a paradox in need of resolution. The paradox is usually presented from the perspective of "landscape theory" [18, where the system is seen as sampling a collection of local minima in a complex potential energy landscape. In this picture the entropy has a configurational contribution that counts the number of accessible minima, and a vibrational contribution associated with the harmonic modes within a representative local minimum. If one makes the reasonable assumption that the vibrational contributions in the glass and crystal are the same, one arrives at the paradox that the glass has fewer configurational states than the crystal. However, we next argue that this partitioning of the entropy is naive and may even be logically inconsistent.

For the "landscape" partitioning of the entropy to be valid, surely the specific heat must be close to the vibrational value $(1 / 4$ in our model) at inverse temperatures above $\beta_{K}$. This value is independent of the strength of the harmonic restoring forces at the local minimum. To claim any other value undercuts the argument that the only relevant modes are vibrations. If one invokes activated "minimum-hopping" to explain the enhanced specific heat (energy fluctuations), then these should be included in the tally of relevant modes along with vibrations. On the other hand, it seems inconsistent to us to invoke such specific heat-boosting modes when cooling to the paradoxical point, and then neglecting them when accounting for the resulting entropy.

The HMM can be helpful in clarifying the mode analysis of the glassy state because we believe we have identified the modes most relevant at low temperatures. These are the rubicons, that is, the elementary transition-state modes whereby the system "crosses" from one basin to another. These modes clearly play a role in boosting energy fluctuations and raising the specific heat above the vibrational 
value. At the same time, because these non-vibrational modes persist to arbitrarily low temperature, it is incorrect to explain the limiting entropy strictly in terms of vibrations. In fact, we see no a priori reason why exotic modes could not have the net effect of bringing the glass entropy below that of the crystal at low temperatures.

It seems reasonable to expect that rubicon modes exist in other models, though perhaps not as transparently. Characterized most broadly, rubicons (i) are elementary modes associated with transition states, (ii) are not thermally activated, and (iii) have a finite density even when the system is quenched to zero temperature. The third property guides us in discovering rubicons in a new system. After a quench one checks whether the system is almost always poised very near a transition state in the energy. It is interesting that empirically the rubicon density in the HMM has an Arrhenius thermal dependence. We do not know whether to expect this to be a general property as well, and in fact we do not understand how this simple behavior arises in the HMM.

How would rubicons manifest themselves in a real network glass? We address this from the perspective of an all-atom molecular dynamics simulator, as this kind of 'experiment' affords the most direct access to the physical processes. Suppose the researcher has prepared the system at temperature $T$ by some cooling protocol, quenches the kinetic energy, and relaxes the system to a configuration of mechanical equilibrium. In the landscape picture, without rubicons, these mechanical equilibria are unremarkable and only weakly sensitive to $T$. The slow dynamics, if kinetic energy were reintroduced, comes about through Arrhenius activation over barriers, as energy gets redistributed through weakly anharmonic processes. With rubicons this picture would be quite different. The mechanical equilibria (after quenching/relaxing) would always be fragile, that is, there would be a finite density of modes (rubicons) with excitation energy $\epsilon$ to a transition state, in the limit $\epsilon \rightarrow 0$. With rubicons around, the system always 'flows', but the rate decreases because the rubicon density is strongly temperature dependent. The last point is important, as our simulations of the HMM brought to light: When the rubicon density is so low that their mean number is less than $O(1)$, the glass will not be in equilibrium. The greatest challenge in realizing equilibrium in all-atom network glass simulations, in the rubicon picture, will therefore be the very large system sizes required, and not so much the long time scales of activated processes.

\section{Quantum model}

To better address the Kauzmann conundrum in the HMM, while also giving a rigorous definition of states and dynamics, we introduce a quantum extension of the model. Whereas the HMM had no parameters, the Hamiltonian of the quantum model now has one:

$$
\mathcal{H}=-\frac{1}{4 N \mu} \Delta_{U}+\Phi(U) .
$$

Here $\Delta_{U}$ is the Laplace-Beltrami operator on $\mathrm{SO}(N)$ and the mass $\mu$ is the sole parameter. Setting the scale factor of the Laplacian so that locally $\left(U=e^{X}, X\right.$ small $)$

$$
\Delta_{U}=\sum_{1 \leq i<j \leq N} \frac{\partial^{2}}{\partial X_{i j}^{2}},
$$

we see, using (5), that the frequency of harmonic oscillation about the Hadamard minima is $\omega=1 / \sqrt{\mu}$. At fixed $\beta$, taking the limit $\mu \rightarrow \infty$ so that $\beta(\hbar \omega) \rightarrow 0$, we 


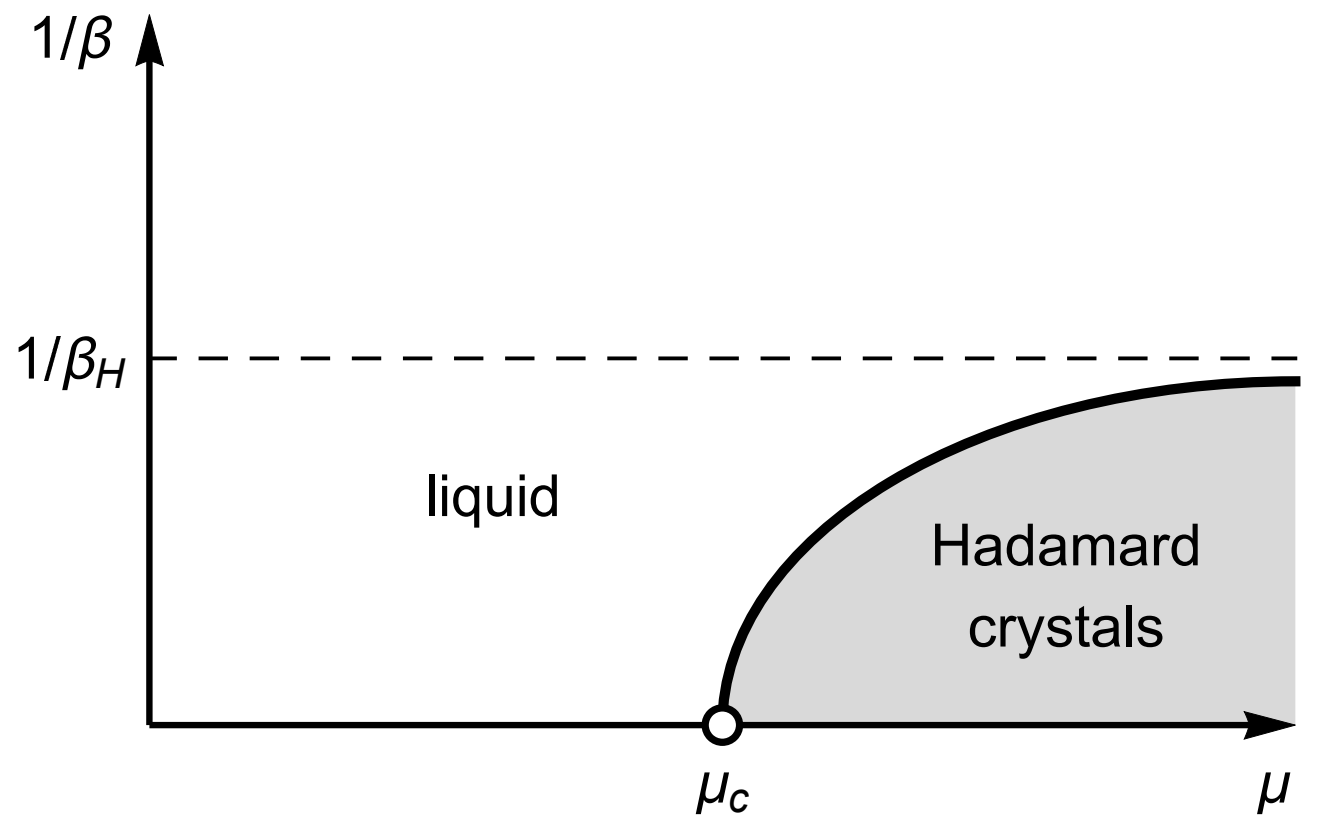

Figure 9. Conjectured equilibrium phase diagram of the quantum hard matrix model.

recover the classical HMM which has a first-order, thermal transition at $\beta=\beta_{H}$. In the $\beta-\mu$ plane we expect this to become a line of first-order transitions. Since $\mu \rightarrow 0$ corresponds to a free particle on $\mathrm{SO}(N)$, a model with no thermal transition, the simplest scenario for the interior of the phase diagram is that the line of firstorder transitions terminates on the zero-temperature axis as sketched in Figure 8 The endpoint of the phase boundary, at $\mu=\mu_{c}$, would then be a quantum (zerotemperature) phase transition. Along the phase boundary the transition state density $\rho_{0}$ drops discontinuously upon crossing into the Hadamard phase.

For investigating quantum equilibrium behavior, the hard matrix model has another advantage over hard spheres in that simulations are a straightforward extension of the classical case. In the standard path-integral scheme, for inverse temperature or imaginary "time" $\beta$ divided into increments $\Delta \beta$, there will be $\beta / \Delta \beta$ classical ensembles, each subject to the potential (1). The only new feature in the quantum simulation is that there is a kinetic contribution,

$$
\operatorname{Tr} U^{T}(\beta) U\left(\beta^{\prime}\right),
$$

for adjacent imaginary times. Sampling the quantum ensemble could still be implemented with bounded-range Givens rotations, now including coupling terms between the two matrices at adjacent times. By contrast, MCMC updates for the quantum hard sphere model require complex world-line reconnections 20 to impose the permutation symmetry of the spheres.

The liquid and Hadamard phases acquire new interpretations when we restrict to the zero temperature axis. In the Hadamard phase the configurations $(U)$ are localized at one of the Hadamard points of $\mathrm{SO}(N)$. In the limit $\mu \rightarrow \infty$ the ladder of excitations becomes more perfectly harmonic and the wave functions have Gaussian decay away 
from these points. On the other side of the quantum phase transition, $\mu<\mu_{c}$, the matrix $U$ is quantum delocalized.

The onset of localization (at a Hadamard point) for $\mu \rightarrow \mu_{c}^{-}$can be detected by measuring the expectation of (26), not just for adjacent times, but for $\left|\beta-\beta^{\prime}\right|$ that span the full range of times in the simulation. While establishing a new quantum phase transitions is a worthwhile end in itself, we should not lose track of the glass phenomenology that motivated the HMM in the first place. In particular, since the $\mu \rightarrow \infty$ limit of the line of first order transitions in Figure 9 is practically non-existent for even moderately large $N$, one may reasonably expect the entire diagram is covered by a single liquid/metastable-glass phase. It is in this setting we should ask what new insights the quantum-HMM can provide.

One way to ask whether quantum mechanics is at all relevant is to compare the order of the limits $\mu \rightarrow \infty$ and $\beta \rightarrow \infty$. On taking the former limit first we get the classical model, and in the previous section we learned that this case brings no surprises. The classical model does not appear to have a dynamical transition: the mixing time grows longer indefinitely, all the way to zero temperature.

The opposite order of limits, where $\mu$ is fixed as we go to zero temperature, brings to mind the phenomenon of Anderson localization [21. For this limit we are interested in the low-lying quantum states of a quantum particle moving on $\mathrm{SO}(N)$ in the presence of a potential that may "look" as random to the particle as an Anderson model. However, before we explore this part of phenomenology we should heed the lesson of many-body localization (MBL) 22 that the temperature of the system, when in isolation, may not be well defined. We therefore need to look for another way to parameterize the transition state density $\rho_{0}$, which, as before, is the core property used by our analysis. As a substitute we use the excitation energy density

$$
\epsilon=\Phi\left(U^{*}\right) / N^{2}+1
$$

of the fragile equilibria $U^{*}$ of the classical model, which satisfy (15). Using the fragile equilibria as a model of the low energy classical states of the glass, where $\epsilon$ is identified with the thermal equilibrium energy density, we expect $\epsilon \sim c_{g} / \beta$, where $c_{g}$ is the low temperature limit of the classical specific heat. Finally, using (17) we arrive at a Lifshitz-tail form for the transition state density:

$$
\rho_{0}(\epsilon) \propto e^{-\epsilon_{0} / \epsilon} .
$$

We are now ready to consider the possibility of an Anderson transition. The method we use is to estimate the fraction of the modes about a fragile equilibrium, characterized by $\epsilon$, that are rubicons rather than phonons. When a suitably large fraction are rubicons, the true quantum states are delocalized. The critical $\epsilon$ above which this happens is analogous to a mobility edge and depends on the mass parameter $\mu$ of the quantum model. When interpreting the result we should remember that $\epsilon$ is only the classical part of the excitation energy.

About each fragile equilibrium $U^{*}$ the potential energy has the form

$$
\Phi\left(X ; U^{*}\right)=\Phi\left(U^{*}\right)+\frac{N}{2} \operatorname{Tr}\left(X^{T} K^{*} X\right)+\cdots,
$$

where

$$
K^{*}=\frac{1}{\sqrt{N}} U^{* T} \operatorname{sgn}\left(U^{*}\right)
$$


is symmetric by (15). Let $V$ be the orthogonal transformation to normal modes $X^{\prime}=V^{T} X V$, where $K^{\prime}=V^{T} K^{*} V$ is diagonal. This does not change the form of the kinetic energy (25). Also, since

$$
\left(K^{*}\right)^{T} K^{*}=\frac{1}{N} \operatorname{sgn}\left(U^{*}\right)^{T} \operatorname{sgn}\left(U^{*}\right)
$$

is approximately the identity matrix (because $\operatorname{sgn}\left(U^{*}\right)$ is nearly Hadamard), so is the diagonalized matrix $K^{\prime}$. Each normal mode coordinate $X^{\prime}$ therefore has zero-phonon wave function

$$
\exp \left(-\sqrt{\mu / 2} N X^{\prime 2}\right)
$$

with amplitude of order

$$
\sqrt{N} \delta X^{\prime} \sim \mu^{-1 / 4}
$$

Finally, since the measure (7) is invariant with respect to the normal mode transformation, so is the density of transition state matrix elements $\rho_{0}$. This gives

$$
f \propto \mu^{-1 / 4} e^{-\epsilon_{0} / \epsilon}
$$

as the fraction of the modes at a fragile equilibrium point that are rubicons, where the proportionality factor is independent of $N$.

If the quantum-HMM has a mobility edge for $\epsilon>\epsilon_{c}$ we speculate it occurs when the fraction $f$ exceeds a critical value, such that modes at all the fragile equilibria are hybridized by rubicons to form extended many-body states. Equation (34) then predicts $\epsilon_{c}$ vanishes with the mass parameter only as

$$
\epsilon_{c} \propto \frac{1}{\log (1 / \mu)},
$$

or that the low energy states do not thermalize when the quantum system is left to itself. This is in contrast to the classical system coupled to a thermal bath, where we saw no evidence of arrested dynamics. As in the classical case, the low energy phenomenology places demands on the thermodynamic limit. In particular, from (33) we see that our mode analysis is well defined only when $N$ becomes suitably large in the limit $\mu \rightarrow 0$ in order to satisfy $\delta X^{\prime} \ll 1$.

\section{Discussion}

The hard matrix model (HMM) challenges the hard sphere model (HSM) in its privileged role of linking the worlds of physics and mathematics on the subject of glass. Both models are highly symmetric and beset with ground states that are largely mysterious, even after over a century of study. As a model of glass, the HSM is considered in the limit of large dimensions [23] in addition to the usual thermodynamic limit of many spheres. The HMM has only the matrix size $N$ in its thermodynamic limit. Without quantum mechanics, by far the most studied case of hard spheres, each model just has one parameter: the packing fraction for the HSM and the temperature for the HMM. The molecular basis for both models - packing of particles (HSM) and covalent bond network (HMM) - is tenuous because the glass arises only in the infinite dimension limit.

Whereas both models are continuum models, the similarity ends already at the level of the elementary modes. The HMM has true vibrations while the HSM requires softening of the spheres (with additional parameters) to exhibit that feature. An 
attractive feature of the HMM is the strong anharmonicity introduced by the cusp in the local energy whenever one of the matrix elements crosses zero. Phonons with significant content of such zero-crossing, called rubicons, are believed to play a key role in the low energy dynamics. Their density is proportional to the density of matrix elements near zero, $\rho_{0}$, a structural property that was found to have a simple Arrhenius behavior.

The observation that $\rho_{0}$ is finite in the thermodynamic limit of the HMM challenges the usual "landscape" picture, where subsystems must receive energy from a bath (the rest of the system) to surmount the energy barriers that carve up the configurations into basins. Rubicons thus provide a mechanism whereby the system can move between very different configurations (matrix elements differing in sign) without activated processes. We believe rubicons are a general feature of glassy systems and credit the HMM for highlighting their existence.

The HMM offers many technical advantages over the HSM in simulations of mechanics, thermodynamics, and even quantum mechanics. This is especially true in the high dimension limit, where keeping track of imminent force discontinuities (at zero-crossings) in the HMM equations of motion is easier than forecasting the next collision event in the HSM. The relative ease of thermodynamic MCMC simulations in the HMM allowed us to directly locate the equilibrium phase transition to the Hadamard phase for sizes up to $N=20$. As far as we know, liquid/crystal equilibrium has not been achieved with MCMC in the HSM in dimensions above three. In the glass phase we found that $N$ had to grow exponentially with the inverse temperature, in experiments up to $N=128$, in order to see thermodynamic limit properties. We are not aware of HSM simulations that go above nine dimensions.

The quantum extensions of the HSM and HMM are candidates for models without disorder that exhibit many-body localization (MBL). It appears there is already a 1D system that fits this description 24. However, there is no consensus on whether such systems might be generic, or whether MBL is at all related to the phenomenology of classical glass. It would be daunting to investigate the spectrum of the quantum-HSM (e.g. for evidence of a level-statistics transition with packing fraction), and nothing close to such a study has ever been attempted. On the other hand, localization for the quantum-HMM Hamiltonian (24) can easily and systematically be investigated by tri-diagonalization [25] applied to a Gaussian basis set with centers sampled near a fragile minimum.

\section{Acknowledgments}

JK-D is supported by NSF Grant No. DMR-1719490. V. E. thanks Persi Diaconis for discussions. The authors thank James Sethna and David Huse for feedback on the manuscript.

\section{Appendix}

This is a derivation of the equation of motion (111) for a particle moving on $\mathrm{SO}(N)$ in the presence of a potential $\Phi(U)$. We start with the Lagrangian

$$
\mathcal{L}=\int d t\left(\frac{\mu N}{2} \operatorname{Tr} \dot{U}^{T} \dot{U}-\Phi(U)+\operatorname{Tr} \Lambda^{T}\left(U^{T} U-1\right)\right),
$$


where all elements of $U$ are treated as independent variables while $\Lambda(t)$, a matrix of Lagrange multipliers, imposes orthogonality on $U$ at all times. Here are the EulerLagrange equations for $U$ :

$$
\mu N \ddot{U}=-\nabla \Phi+U\left(\Lambda+\Lambda^{T}\right)
$$

The first term on the right hand side is just the matrix of partial derivatives of $\Phi$. Here is the transpose of the same set of equations:

$$
\mu N \ddot{U}^{T}=-(\nabla \Phi)^{T}+\left(\Lambda+\Lambda^{T}\right) U^{T} .
$$

Next, take one and two time derivatives of the constraint $1=U^{T} U$ to get two additional constraint equations:

$$
\begin{aligned}
& 0=\dot{U}^{T} U+U^{T} \dot{U} \\
& 0=\ddot{U}^{T} U+2 \dot{U}^{T} \dot{U}+U^{T} \ddot{U} .
\end{aligned}
$$

Now multiply A.2 on the left by $U^{T}$, A.3 on the right by $U$, add the result and use (A.5) to eliminate the double time derivatives:

$$
-\mu N \dot{U}^{T} \dot{U}=-\frac{1}{2} U^{T}(\nabla \Phi)-\frac{1}{2}(\nabla \Phi)^{T} U+\Lambda+\Lambda^{T} .
$$

Multiply this equation on the left by $U$ and take the resulting expression for $U\left(\Lambda+\Lambda^{T}\right)$ so obtained and substitute into (A.2) to eliminate the Lagrange multipliers:

$$
\mu N\left(\ddot{U}+U \dot{U}^{T} \dot{U}\right)=-\frac{1}{2}\left(\nabla \Phi-U(\nabla \Phi)^{T} U\right) .
$$

After using (A.4) to show $U \dot{U}^{T} \dot{U}=\dot{U} \dot{U}^{T} U$, we arrive at equation (11).

\section{References}

[1] Randall D Kamien and Andrea J Liu. Why is random close packing reproducible? Physical Review Letters, 99(15):155501, 2007.

[2] LF Cugliandolo, J Kurchan, Giorgio Parisi, and F Ritort. Matrix models as solvable glass models. Physical review letters, 74(6):1012, 1995.

[3] Marin Soljačić and Frank Wilczek. Minimal potentials with very many minima. Physical review letters, 84(11):2285, 2000.

[4] A. Crisanti and H. J. Sommers. The spherical $p$-spin interaction spin glass model: the statics. Zeitschrift für Physik B Condensed Matter, 87(3):341-354, 101992.

[5] A. Crisanti, H. Horner, and H. J. Sommers. The spherical $p$-spin interaction spin-glass model. Zeitschrift für Physik B Condensed Matter, 92(2):257-271, 61993.

[6] Tommaso Castellani and Andrea Cavagna. Spin-glass theory for pedestrians. Journal of Statistical Mechanics: Theory and Experiment, 2005(05):P05012, 52005.

[7] A Hedayat, Walter Dennis Wallis, et al. Hadamard matrices and their applications. The Annals of Statistics, 6(6):1184-1238, 1978.

[8] Thomas C Hales. A proof of the Kepler conjecture. Annals of Mathematics, pages 1065-1185, 2005.

[9] H Kharaghani and B Tayfeh-Rezaie. On the classification of Hadamard matrices of order 32. Journal of Combinatorial Designs, 18(5):328-336, 2010.

[10] N J A Sloane and Simon Plouffe. Online Encyclopedia of Integer Sequences, https://oeis.org/A206711, 2019.

[11] Warwick de Launey and Daniel M Gordon. On the density of the set of known Hadamard orders. Cryptography and Communications, 2(2):233-246, 2010.

[12] Siun-Chuon Mau and David A Huse. Stacking entropy of hard-sphere crystals. Physical Review E, 59(4):4396, 1999.

[13] F. W. Ponting and H. S. A. Potter. The Volume of Orthogonal and Unitary Space. The Quarterly Journal of Mathematics, os-20(1):146-154, 011949.

[14] Helmut G Katzgraber, Simon Trebst, David A Huse, and Matthias Troyer. Feedback-optimized parallel tempering Monte Carlo. Journal of Statistical Mechanics: Theory and Experiment, 2006(03):P03018-P03018, 32006. 
[15] J C Phillips. Topology of covalent non-crystalline solids I: Short-range order in chalcogenide alloys. Journal of Non-Crystalline Solids, 34(2):153-181, 1979.

[16] Persi Diaconis. Patterns in eigenvalues: the 70th Josiah Willard Gibbs lecture. Bulletin of the American Mathematical Society, 40(2):155-178, 2003.

[17] Mark D Ediger, C Austen Angell, and Sidney R Nagel. Supercooled liquids and glasses. The Journal of Physical Chemistry, 100(31):13200-13212, 1996.

[18] Pablo G Debenedetti and Frank H Stillinger. Supercooled liquids and the glass transition. Nature, 410(6825):259, 2001.

[19] Walter Kauzmann. The nature of the glassy state and the behavior of liquids at low temperatures. Chemical Reviews, 43(2):219-256, 1948.

[20] David M Ceperley and EL Pollock. Path-integral computation of the low-temperature properties of liquid He 4. Physical Review Letters, 56(4):351, 1986.

[21] Philip W Anderson. Absence of diffusion in certain random lattices. Physical Review, 109(5):1492, 1958.

[22] Rahul Nandkishore and David A Huse. Many-body localization and thermalization in quantum statistical mechanics. Annu. Rev. Condens. Matter Phys., 6(1):15-38, 2015.

[23] Patrick Charbonneau, Jorge Kurchan, Giorgio Parisi, Pierfrancesco Urbani, and Francesco Zamponi. Glass and jamming transitions: From exact results to finite-dimensional descriptions. Annual Review of Condensed Matter Physics, 8:265-288, 2017.

[24] James M Hickey, Sam Genway, and Juan P Garrahan. Signatures of many-body localisation in a system without disorder and the relation to a glass transition. Journal of Statistical Mechanics: Theory and Experiment, 2016(5):054047, 2016.

[25] Roger Haydock. The electronic structure of weak, random, two-and three-dimensional potentials. Philosophical Magazine B, 43(2):203-218, 1981. 